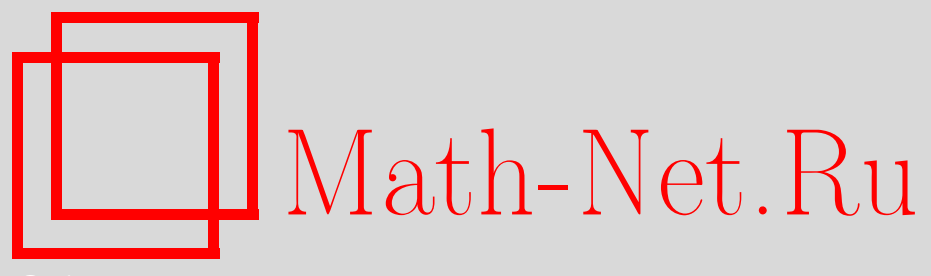

Ф. А. Устинов, Асимптотика среднего времени запаздывания в задаче о разладке для базисных процессов Леви в обобщенной байесовской постановке, УМН, 2009, том 64, выпуск 1, 161-162

DOI: https://doi.org/10.4213/rm9275

Использование Общероссийского математического портала Math-Net.Ru подразумевает, что вы прочитали и согласны с пользовательским соглашением http://www . mathnet.ru/rus/agreement

Параметры загрузки:

IP: 54.80 .73 .141

26 апреля 2023 г., $15: 21: 48$

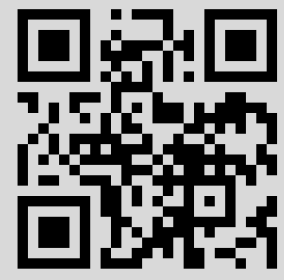




\section{Асимптотика среднего времени запаздывания в задаче о разладке для базисных процессов Леви в обобщенной байесовской постановке}

\section{Ф. А. Устинов}

1. Пусть на некотором вероятностном пространстве $(\Omega, \mathscr{F}, \mathrm{P})$ наблюдается процесс $X=\left(X_{t}\right)_{t \geqslant 0}: X_{t}=\int_{0}^{t} I\{s<\theta\} d X_{s}^{(0)}+\int_{0}^{t} I\{s \geqslant \theta\} d X_{s}^{(1)}$, где $X_{t}^{(i)}=\sigma B_{t}^{(i)}+\mu_{i} t+\sum_{k=0}^{N^{i}} \xi_{k}^{(i)}$, $i=0,1$. Здесь $B^{(i)}$ - процессы броуновского движения, $N^{i}$ - пуассоновские процессы интенсивности $\lambda_{i}, \xi_{k}^{(0)}$ и $\xi_{j}^{(1)}$ - наборы независимых случайных величин, имеющих эквивалентные распределения $F^{(0)}$ и $F^{(1)}$ соответственно. Случай $\sigma \neq 0$ будем называть

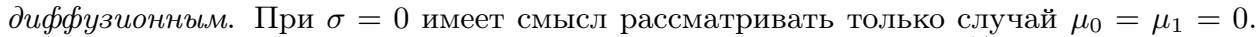
Этот случай будем называть недиффузионным. Процессы типа $X^{(i)}$ играют базисную роль при построении процессов Леви. Неслучайный момент $\theta \in[0,+\infty]$ интерпретируется как момент разладки, или смены режима процессом $X$.

В заметке рассматривается ряд вопросов, связанных с нахождением оптимального момента остановки, приближающего $\theta$ (определение оптимальности дано ниже).

Обозначим $P_{\theta_{0}}$ распределение процесса $X$ в предположении, что момент разладки $\theta$ равен $\theta_{0}$. При распределении $P_{\infty}$ разладка не происходит никогда, при $P_{0}$ разладка происходит мгновенно, в момент времени 0. Момент подачи сигнала тревоги об обнаружении разладки обозначим $\tau$. Это марковский момент относительно естественной фильтрации $\left\{\mathscr{F}_{t}^{X}\right\}_{t \geqslant 0}$, порожденной процессом $X$. Следуя [1], будем искать наилучший момент в классе $\widetilde{\mathfrak{M}}_{T}=\left\{\tau: E_{\infty} \tau \geqslant T\right\}$, т. е. среди правил остановки, для которых среднее время до подачи (ложной) тревоги в случае отсутствия разладки не меньше $T$. Момент $\tau^{*}$ будем называть оптимальным, если величина $B(T):=\frac{1}{T} \inf _{\tau \in \mathfrak{M}_{T}} \int_{0}^{\infty} E_{\theta}(\tau-\theta)^{+} d \theta$, называемая риском, а также средним временем запаздывания, достигается на $\tau^{*}$. В этом критерии $\theta$ можно интерпретировать как обобщенную случайную величину с равномерным распределением на $[0, \infty]$.

Для нахождения оптимального момента рассмотрим процесс Ширяева (см. [2]): $\psi_{t}=\psi_{0} L_{t}+\int_{0}^{t} \frac{L_{t}}{L_{s}} d s$, где $L_{t}=\frac{d\left(P_{0} \mid \mathscr{F}_{t}^{X}\right)}{d\left(P_{\infty} \mid \mathscr{F}_{t}^{X}\right)}-$ производная Радона-Никодима сужения меры $P_{0}$ на сигма-алгебру, порожденную процессом $X$ до момента $t$, по сужению меры $P_{\infty}$ на ту же сигма-алгебру. При помощи статистики $\psi$, почти дословно повторяя рассуждения из [1; лемма 2.1], можно получить представление $B(T)$ в виде

$$
B(T)=\frac{1}{T} \inf _{\tau \in \mathfrak{\mathfrak { M }}_{T}} E_{\infty}^{0} \int_{0}^{\tau} \psi_{s} d s
$$

Здесь и ниже $E^{x}(\cdot)$ означает $E\left(\cdot \mid \psi_{0}=x\right)$. В случае $x=0$ индекс будем опускать. При помощи метода ("множителей Лагранжа") из [1; раздел 4] и анализа траекторий процесса $\psi$ получаем следующий результат.

Теорема 1. Оптимальный момент в классе $\widetilde{\mathfrak{M}}_{T}-$ это момент $\tau_{A}$ первого выхода прочесса $\psi_{t}$ на уровень $A=A(T)$ такой, что $E_{\infty} \tau_{A}=T$.

Результат верен и для процессов $X$, в определении которых процессы $X^{(1)}, X^{(2)}$ заменены на два произвольных процесса Леви с эквивалентным распределением.

2. Интересно значение функции риска $B(T)$ при больших $T$ (в том числе как оценка в соответствующей минимаксной задаче, см. [1]). Чтобы упростить запись, мы рассмотрим только 2 случая: когда в выражении для $X$ параметры $\left(\sigma, \mu_{0}, \mu_{1}\right)$ равны $(0,0,0)$ и когда $\left(\sigma, \mu_{0}, \mu_{1}\right)=(1,0, \sqrt{2})$. Остальные случаи рассматриваются 
аналогично. Асимптотику $B(T)$ при $T \rightarrow \infty$ можно вычислить, действуя следую-

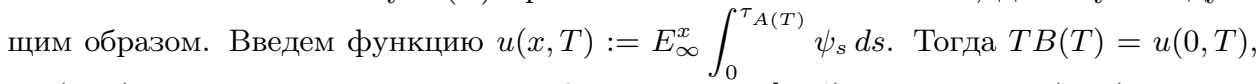
а $u(x, T)$ удовлетворяет уравнению $\mathscr{L} u=-x, x \in[0, A)$, и условию $u(x, T)=0$ при $x \geqslant A$ [3; гл. 3]. Здесь $\mathscr{L}$ - инфинитезимальный оператор процесса $\psi$, имеющий вид

$\mathscr{L} u=\left(1-\delta_{0 \sigma}\right) x^{2} u^{\prime \prime}(x)+\left(1+\left(\lambda_{0}-\lambda_{1}\right) x\right) u^{\prime}(x)+\lambda_{0}\left[\int_{\mathbb{R}} u\left(x \frac{\lambda_{1}}{\lambda_{0}} f(r)\right) d F^{(0)}(r)-u(x)\right]$, где $f(r)=\frac{d F^{(1)}}{d F^{(0)}}(r), \delta_{i j}$ - символ Кронекера (см. в [4; гл. 3] стохастическое дифференциальное уравнение для $L_{t}$, из него выводится соответствующее уравнение для $\psi$, затем выражение для оператора $\mathscr{L}$ ). Верна следующая теорема.

Теорема 2. Непрерывное в точке А решение задачи

$$
\mathscr{L} v(x)=h(x), \quad 0 \leqslant x<A, \quad v(x)=0, \quad x \geqslant A,
$$

где $h(x)$ - непрерывная на $[0, A)$ функиия, единственно.

Теорема позволяет получать аппроксимации для $B(T)$. Общая схема такова. Подбирается некоторая функция $\tilde{u}(x)$ такая, что $\mathscr{L} \tilde{u}(x)=-x+o(x), 0 \leqslant x<A$, и $\tilde{u}(x)=0, x \geqslant A$. С помощью теоремы можно показать, что функция $\tilde{u}(x)$ будет близка к $u(x)$.

Таким образом, задача сводится к нахождению функции $\tilde{u}(x)$. В аналитически вычислимом случае разладки для броуновского движения получается функция $x \log x$. В произвольном случае аналитическое решение невозможно, но главный член разложения приближения решения $(\tilde{u}(x))$ имеет такой же порядок, как точное решение в броуновском случае. Обозначим

$$
C=\left(\lambda_{0}-\lambda_{1}+\lambda_{0} \int_{\mathbb{R}} \frac{\lambda_{1}}{\lambda_{0}} f(r) \log \left(\frac{\lambda_{1}}{\lambda_{0}} f(r)\right) d P_{0}(r)\right)^{-1}, \quad D=\left(1+C^{-1}\right)^{-1} .
$$

В случае направленных вниз скачков процесса $\psi$ имеет место следующая

Teopema 3. Ecлu $\left(\sigma, \mu_{0}, \mu_{1}\right)=(0,0,0)$, mo

$$
B(T)=C \log T+o(\log T) \quad \text { nрu } T \rightarrow \infty .
$$

$\operatorname{Eсли~}\left(\sigma, \mu_{0}, \mu_{1}\right)=(1,0, \sqrt{2})$, mo

$$
B(T)=D \log T+o(\log T) \quad \text { при } T \rightarrow \infty .
$$

Для случая произвольных скачков можно найти связь главного члена асимптотики и математического ожидания времени выхода на уровень $A$ :

Teоpema 4. Ecлu $\left(\sigma, \mu_{0}, \mu_{1}\right)=(0,0,0)$, mo

$$
B(T)=C E_{\infty} \tau_{A} \cdot T^{-1} \log T+o(\log T) \quad \text { nрu } T \rightarrow \infty .
$$

$\operatorname{Ecлu}\left(\sigma, \mu_{0}, \mu_{1}\right)=(1,0, \sqrt{2})$, mo

$$
\begin{array}{r}
B(T)=D E_{\infty} \tau_{A} \cdot T^{-1} \log T+o(\log T) \quad n p u T \rightarrow \infty . \\
\text { Список литературы }
\end{array}
$$

[1] E. А. Feinberg, А. N. Shiryaev, Statist. Decisions, 24:4 (2006), 445-470. [2] А. Н. Ширяев, Теория вероятн. и ее примен., 8:1 (1963), 26-51. [3] G. Peskir, A. N. Shiryaev, Optimal stopping and free-boundary problems, Birkhäuser, Basel, 2006. [4] Ж. Жакод, А. Н. Ширяев, Предельные теоремы для случайных процессов, т. I, Теор. вероятн. и матем. стат., 47, Наука, М., 1994.

Ф. А. Устинов (Ph. А. Ustinov)

Московский государственный университет им. М. В. Ломоносова

E-mail: atikin85@mail.ru
Представлено А. В. Булинским Принято редколлегией 07.12 .2008 